\title{
Crystal structure of Cry51Aa1: A potential novel \\ insecticidal aerolysin-type $\beta$-pore-forming toxin from \\ Bacillus thuringiensis
}

\section{Chengchen $\mathrm{Xu}^{\mathrm{a}, \mathrm{b}}$, Unmesh Chinte ${ }^{\mathrm{b}, \mathrm{c}}$, Lirong Chen ${ }^{\mathrm{b}}$, Qingqing Yao ${ }^{\mathrm{d}}$, Ying Meng ${ }^{a}$, Dayong Zhou ${ }^{b}$, Li-Jun Bi ${ }^{e}$, John Rose ${ }^{b, c}$, Michael J. Adang ${ }^{b}$, , Bi- Cheng Wang ${ }^{\mathrm{b}, \mathrm{c}^{*}}$, Ziniu $\mathrm{Yu}^{\mathrm{a}}{ }^{*}$, and Ming Sun ${ }^{\mathrm{a} *}$}

${ }^{a}$ State Key Laboratory of Agricultural Microbiology, College of Life Science and Technology, Huazhong Agricultural University, Wuhan, Hubei, 430070, P. R. China ${ }^{b}$ Department of Biochemistry and Molecular Biology, University of Georgia, Athens, GA, 30602, USA

'SER-CAT, University of Georgia and the Advanced Photon Source, Argonne National Laboratory, Argonne, IL, 60439, USA

${ }^{\mathrm{d} D e p a r t m e n t}$ of Biochemistry, School of Life Sciences, Fudan University, Shanghai, 200438, P. R. China

${ }^{\text {e}}$ National Laboratory of Biomacromolecules, Institute of Biophysics, Chinese Academy of Sciences, Beijing, 100101, P. R. China

fDepartment of Entomology, University of Georgia, Athens, GA, 30602, USA

*Correspondence email: wang@bcl1.bmb.uga.edu; yz41@mail.hzau.edu.cn; m98sun@mail.hzau.edu.cn 


\section{Abstract}

The structures of several Bacillus thuringiensis (Bt) insecticidal crystal proteins have been determined by crystallographic methods and a close relationship has been explicated between specific toxicities and conserved three-dimensional architectures. In this study, as a representative of the coleopteran- and hemipteran-specific Cry51A group, the complete structure of Cry51Aa1 protoxin has been determined by X-ray crystallography at $1.65 \AA$ resolution. This is the first report of a coleopteran-active Bt insecticidal toxin with high structural similarity to the aerolysin-type $\beta$-pore forming toxins ( $\beta$-PFTs). Moreover, study of featured residues and structural elements reveal their possible roles in receptor binding and pore formation events. This study provides new insights into the action of aerolysintype $\beta$-PFTs from a structural perspective, and could be useful for the control of coleopteran and hemipteran insect pests in agricultures.

Key words: Bacillus thuringiensis, crystal structure, insecticidal, pore-forming toxin 


\section{Introduction}

Bacillus thuringiensis (Bt), a species of Gram-positive soil bacterium, produces parasporal crystal toxins having insecticidal properties during sporulation [1]. By functional and structural comparisons, Bt toxins can be categorized into two major families, Crystal (Cry) and Cytolytic (Cyt) toxins [2]. The structures of nine Cry and three Cyt toxins have been determined and show a conserved structural architectures based on their class (Cry or Cyt) and their distinct insecticidal mechanisms $[3,4]$.

Recently, a new group of Bt toxins, called parasporins, has been identified with the unique features of being non-insecticidal, but having cytocidal activity against human cancer cells [5]. Structures of parasporin-2 (PDB ID 2ZTB) and a 26-kDa nontoxic protein 26NPT (PDB ID 2D42) have been determined. Distinct from Cry and Cyt toxins, parasporin-2 and 26NPT exhibit striking structural similarity to the aerolysin-type $\beta$-pore-forming toxins (aerolysin-type $\beta$-PFTs) $[6,7]$. Despite aerolysin-type $\beta$-PFTs coming from diverse species and the limited sequence identities within the family, their structural similarities, especially the conserved $\beta$ strands topology, suggest a common mode of action.

While the three-dimensional structures of many Bt parasporal crystal toxins can be grouped into categories of specific toxicities, there are exceptions. Some parasporins with strong cytotoxicities against cancer cells (e.g. parasporin-1, parasporin-3, and parasporin-6) are structurally similar to the insecticidal threedomain type Cry toxins. In contrast, the newly released Cry34Ab1 (PDB ID 4JOX) and Cry35Ab1 (PDB ID 4JP0) structures have respective similarities to aegerolysin 
and aerolysin-like toxins; yet the Cry34/35 toxin complex has insecticidal toxicity against corn rootworms [8].

The Cry51A toxins are examples of Cry toxins with unusual insecticidal specificities and structures distinct from the three-domain type architectures commonly observed. In this study, we present the precise X-ray crystallographic structure of Cry51Aa1 at $1.65 \AA$ resolution and establish its toxicity to coleopteran larvae. Structural features not only demonstrate that this coleopteran-active insecticidal toxin has significant structural similarity to the aerolysin-type $\beta$-PFTs, but reveal its possible mode of action in midgut membrane.

\section{Materials and Methods}

\subsection{Expression, solubilization and purification}

Cry51Aa1 (GeneBank QD836184.1) was identified in Bt strain F14-1, which was isolated from a soil sample collected in northern China [9]. Full length protoxin was expressed in Bt acrystalliferous host strain BMB171, using E.coli-Bacillus shuttle vector $\mathrm{pHT} 304$ [10]. The recombinant Bt strain was cultured at $30{ }^{\circ} \mathrm{C}$ in tryptic soy broth medium for $4 \mathrm{~d}$. After sporulation, cells were harvested and washed with $1 \mathrm{M}$ $\mathrm{NaCl}$ and distilled water respectively. Then, the protoxin was solubilized in $20 \mathrm{mM}$ $\mathrm{Na}_{2} \mathrm{CO}_{3}$ buffer, pH 10 containing $20 \mathrm{mM}$ EDTA at room temperature for $3 \mathrm{~h}$.

Solubilized Cry51Aa1 was filtered by a $0.2 \mu \mathrm{m}$ filter (Millipore), and purified by ion-exchange chromatography on a $5 \mathrm{~mL}$ HiTrap Q HP column (GE Healthcare), preequilibrated with $20 \mathrm{mM} \mathrm{Na}{ }_{2} \mathrm{CO}_{3}$ buffer, $\mathrm{pH}$ 10. Cry51Aa1 was eluted using a $\mathrm{NaCl}$ gradient from 0 to $500 \mathrm{mM}$ in the same buffer. The eluted protoxin was concentrated in an Amicon Ultra-15 10,000 MWCO centrifugal filter (Millipore) and 
further purified by gel filtration chromatography applied with Superdex 200 16/60 column (GE Healthcare), pre-equilibrated with $20 \mathrm{mM}$ Glycine- $\mathrm{NaOH}, \mathrm{pH} 10$. The fractions containing Cry51Aa1 were collected and concentrated to $3 \mathrm{mg} / \mathrm{ml}$ for crystallization.

\subsection{Crystallization and data collection}

Crystallization trials were carried out with the sitting-drop vapor diffusion method. The $20 \mu \mathrm{l}$ drops were prepared by a $3: 1$ mixture of $0.5 \mathrm{mg} / \mathrm{ml}$ protein solution and the reservoir solution containing $0.14 \mathrm{M}\left(\mathrm{NH}_{4}\right)_{2} \mathrm{SO}_{4}, 0.1 \mathrm{M} \mathrm{CH} \mathrm{COONa}_{3}$ $(\mathrm{pH} 3)$ and $16 \% \mathrm{w} / \mathrm{v}$ polyethylene glycol 2000 . Setups were then incubated at $18{ }^{\circ} \mathrm{C}$

- Diamond shaped crystals formed and grew to a maximum dimension of $0.4 \times 0.2 \times 0.1 \mathrm{~mm}^{3}$ in three weeks. To obtain derivative diffraction data, native crystals were incubated for $1 \mathrm{~h}$ at $18{ }^{\circ} \mathrm{C}$ in a $25 \mathrm{mM} \mathrm{K}_{2} \mathrm{O}_{\mathrm{S}} \mathrm{Cl}_{6}$ solution prepared using the equilibrated reservoir solution that produced the crystal. Crystals were flashfrozen in liquid nitrogen after cryoprotection, with $20 \% \mathrm{v} / \mathrm{v}$ glycerol. Data for both native and derivative crystals were collected on beamline 22-BM, SER-CAT, Advanced Photon Source, Argonne National Laboratory. Diffraction images were indexed, integrated and scaled using HKL2000 [11].

\subsection{Structure determination}

The structure of Cry51Aa1 was determined by the single-wavelength anomalous diffraction (SAD) method using $2.8 \AA$ resolution data set collected from an osmium soaked crystal. Initial calculation were carried out using Phenix [12]. The Phenix.Autosol solution fit $\sim 65 \%$ of the amino acid chain. Molecular replacement 
was then used to orientate the partial model in the high-resolution (1.65 $\AA$ ) native cell. Auto-Richshaw [13], followed by manual correction using COOT [14] was then used to complete the model. The final $1.65 \AA$ Phenix refined model contains residues 5-309, 443 solvent molecules modeled as water. Residues 1-4 are lacking of supporting electron density and presumed to be disordered. Cry51Aa1 crystallized as a monomer in the asymmetric unit corresponding to a calculated [15] solvent content of $\sim 47 \%$. Details for data collection, structure solution and refinement are summarized in Table S1. The coordinates and structure factors for Cry51Aa1 have been deposited in the Protein Data Bank with the PDB ID 4PKM

\subsection{Insecticidal assays}

Beetle insecticidal assays were carried out against Leptinotarsa decemlineata (Colorado potato beetle, CPB) neonates. As the control, Cry3Aa from $B$. thuringiensis subsp. tenebrionis was expressed and solubilized as described for Cry51Aa1. CPB eggs were purchased from French Agricultural Research, Inc. Red Pontiac potato seeds were obtained from a local farmers' market. CPB bioassays were performed according to a Cry3Aa bioassay described previously; the exception being that toxin materials were applied by a leaf-dipping method [16]. Toxin solubilization buffer ( $\left.20 \mathrm{mM} \mathrm{Na} \mathrm{CO}_{3}, \mathrm{pH} 10\right)$ was used as the negative control. Potato leaves were dipped for $30 \mathrm{~s}$ with concentrations of Cry51Aa1 and Cry3Aa ranging from 5 to $100 \mu \mathrm{g} / \mathrm{ml}$ and 0.05 to $100 \mu \mathrm{g} / \mathrm{ml}$, respectively. Each concentration was treated in triplicate with 30 CPB larvae (5 larvae per 1 oz. plastic cup). After incubating at $28{ }^{\circ} \mathrm{C}$ for $5 \mathrm{~d}$, concentrations of Cry51Aa 1 and Cry3Aa that 
resulted in $50 \%$ mortality were calculated by Probit analysis using SPSS software [17].

\section{Results and Discussion}

\subsection{Crystal structure of Cry51Aa1 monomer}

The structure of Cry51Aa1 (Fig. 1) is elongated at dimensions of approximately $96 \AA \times 30 \AA \times 25 \AA$, with a total surface area of $15,418 \AA^{2}[18]$. Secondary structure components include five helices and fifteen $\beta$-strands. Helices a1-a4 are located in the $\mathrm{N}$-terminal domain of the molecule while helix a5 is located close to the $\mathrm{C}$ terminus of the protein. The long $\beta$-strands observed in the structure are apparently the most striking features of the molecule with some strands (strands $\beta 5, \beta 9$ and $\beta 12$ ) having lengths of over $68 \AA$. The molecule has a similar topology to other $\beta$-PFTs structures described previously and share their three-domain architecture [6].

Domain I (residues 5-46 and 198-236) consists of two discontinuous parts. Part one begins with strand $\beta 1(6-8)$ (note residues in parentheses) which is closely followed by a-helix a1 (9-27) and two short $3_{10}$-helices, a2 (39-41) and a3 (44-46). The largest a-helix, a1, extends approximately $22 \AA$ from $\mathrm{N}$-terminus to the center of Domain I. Part two is made up of a short $3_{10}$-helice a4 (223-226) and an antiparallel $\beta 10(205-210), \quad \beta 11(217-221) \quad \beta$-hairpin. By slightly distorted in a counterclockwise direction, a large hydrophobic core of 24 residues is buried within these two parts.

Domain II (residues 47-65, 94-156, 181-197, and 237-258) forms a $\beta$-sandwich composed of one anti-parallel five-stranded $\beta$-sheet $(\beta 2(47-50) / \beta 3(53-55), \beta 5$ 
(94-106), $\beta 8$ (151-156), $\beta 9$ (181-196), and $\beta 12$ (244-258)) and one antiparallel $\beta$ hairpin ( $\beta 6(115-126)$ and $\beta 7$ (132-143)). Strands $\beta 2$ and $\beta 3$ are connected by a two-residue bridge to form an extended $\beta$ strand. Adjacent and running parallel to the extended strand $\beta 2 / \beta 3$, the antiparallel $\beta$-hairpin twists at least $80^{\circ}$ and runs diagonally for about $52 \AA$ across the five-stranded $\beta$-sheets. The $\beta 6-\beta 7$ hairpin is amphipathic with its hydrophobic side facing towards the molecule and its hydrophilic side facing solvent.

Domain III (residues 66-93, 157-180, and 259-309) is the C-terminal domain. It also contains a $\beta$-sandwich comprised of a three-stranded ( $\beta 4$ (66-77), $\beta 9$ (171179), and $\beta 12$ (259-268)) and a two-stranded ( $\beta 5$ (83-93) and $\beta 8(157-167)) \beta-$ sheet, with an extra pair of strands $\beta 13(275-280) / \beta 14(283-285)$ that are joined by a short 2-residue bridge. Strands $\beta 14$ and $\beta 15$ (291-293) form a $\beta$-hairpin, followed by a short $33_{10}$-helix a5 (294-296), and a long 13 -resiude unstructured 'tail' composed at the C-terminus that is involved in dimer stabilization.

To determine the structural similarity between Cry51Aa1 and other aerolysintype $\beta$-PFTs a DALI search [19] for structural homologues was carried out. The closest DALI homologues having $Z$ scores $>10$ are: (1) the nontoxic 26NPT (PDB ID 2D42, Z score=17.9, RMSD $2.4 \AA$ ); (2) the pathogenic epsilon (PDB ID 1UYJ, Z score=12.5, RMSD 4.6 $\AA$ ). For comparison, the hemolytic LSL (PDB ID 1W3F) gave a $Z$ score $=8.9$ and an RMSD $4.0 \AA$; the cytolytic aerolysin (PDB ID 1PRE) gave a Z score $=7.6$ and an RMSD of $6.8 \AA$; and the anti-cancer parasporin-2 (PDB ID 2ZTB) gave a $Z$ score $=7.2$ with an RMSD of $5.4 \AA$. Although sequence identities between Cry51Aa1 and these proteins are relatively low ( $8 \%-18 \%)$, the $\beta$-stranded domain structure presents a strikingly conserved structural homology (Fig. 2A). 


\subsection{Intramolecular interactions at domain boundary}

The Domain I-Domain II interface is stabilized by a salt bridge formed between Asp195 ( $\beta 9)$ and His 199 ( $\beta 9-\beta 10$ loop) and extensive hydrogen bonding. Hydrogen bonds connect strand $\beta 1$ and helix $a 1$ to strand $\beta 5$; also connect helix $a 4$ and the C-terminal end of strand $\beta 10$ to strands $\beta 12, \beta 2$ and $\beta 9$, with the $\beta 9-\beta 10$ loop serving as a 'bridge' between Domain I and Domain II (Figure S1). At Domain IIDomain III interface, the amphipathic $\beta 6-\beta 7$ hairpin interacts with both the $\beta 3-\beta 4$ loop of domain II and helix a5 and the C-terminal tail of Domain III, and the surrounding hydrogen bonds of the elongated $\beta 6-\beta 7$ loop could reinforce intramolecular interactions at this boundary and contribute to the molecule stabilization (Fig. 3E). The observations of intramolecular interactions are consistent with crystallographic B-factors (Fig. 3C). Distribution of B-factors reveals that inter-domain boundaries have the lowest B values, while high B-factors are observed for atoms associated with the flexible loops.

\subsection{Cry51Aa1 dimer in the crystal}

Cry51Aa1 crystal structure has one molecule in the asymmetric unit but two-fold crystal symmetry generates a crystallographic head-to-tail dimer (Fig. 3A), with Domain I of monomer 1 interacting with Domain III and the C-terminal tail of monomer 2. When viewed perpendicular to the dimer's two-fold axis, the two monomer across each other at $\sim 30^{\circ}$ angle (Fig. 3B). The dimer is slightly longer than the Cry51Aa1 monomer with dimensions of approximately $100 \AA \times 55 \AA \times 52 \AA$. The PISA [18] analysis shows that the monomer-monomer interface is extensive with the area an estimated buried surface area of $1,972 \AA^{2}$ per molecule, or 
$\sim 12.7 \%$ of the monomer surface. There is a solvent-accessible channel at the center of the dimer with solvent-exposed area covering about 2,000 $\AA^{2}$, or approximately $7 \%$ of the total solvent-accessible area of the dimer. The dimer interface is stabilized by over 30 pairs of hydrogen bonds (Fig. 3D and Table S2).

\subsection{Cry51A is an insecticidal toxin group with coleopteran specificity}

Cry51Aa1 and Cry51Aa2 toxins share $97.7 \%$ sequence identity (Fig. 2B). By sequence alignment, Cry51Aa1 has extra three residues of ${ }^{1} \mathrm{MIF}^{3}$ at its $\mathrm{N}$ terminus. Meanwhile, Cry51Aa2 has an additional 'HYS' fragment between residues Glu198 and His199 (Cry51Aa1 numbering) in a solvent exposed area of the $\beta 9-\beta 10$ loop located at the interface of Domain I and Domain II.

Cry51Aa2 has insecticidal toxicity against CPB, Lygus hesperus, and Lygus lineolaris, and has been successfully expressed in transgenic cotton plants conferring anti-hemipteran activity [20]. It was reported that the mortality against CPB neonates was about $68.45 \%$ when the concentration of Cry51Aa2 was at 0.5 $\mathrm{mg} / \mathrm{ml}$, in a diet incorporation bioassay [21]. To establish the toxicity of Cry51Aa1, CPB neonates were fed with Cry51Aa1 protoxin by leaf-dipping method, using the Cry3Aa protoxin as control. The $\mathrm{LC}_{50}$ of Cry51Aa1 against CPB was $19.5 \mu \mathrm{g} / \mathrm{ml}$, which was less than the $\mathrm{LC}_{50}$ of Cry3Aa at $0.669 \mu \mathrm{g} / \mathrm{ml}$ (Table S3). The insecticidal assays of Cry51Aa1 against CPB support the toxicity preferences of the Cry51A group for coleopteran larvae. Moreover, although Cry51A toxins and the threedomain type Cry3 toxins both have toxicities to coleopteran larvae, based on their structural dissimilarity, it is likely that they recognize different midgut receptors in larvae and have distinct modes of action. 


\subsection{Putative roles of aromatic residues in Domain I}

In aerolysin-type PFTs, aromatic residues are abundant in Domain I of parasporin-2, epsilon and LSL, and in Domain I and II of aerolysin. It has been reported that Domain II of aerolysin can recognize the GPI-anchor backbone (ethanolamine- $\mathrm{HPO}_{4}-6$ Mana1-2Mana1-6Mana1-4GlcNH${ }_{2} \mathrm{a} 1-6-m y o-i n o s i t o l-1 \mathrm{HPO}_{4}$ ), while Domain I is involved in binding to an N-linked glycan complex on the GPIanchored receptor [22]. Mutations to residues Trp34, Trp45, and Tyr61 in Domain I, Tyr162, Trp324, and His332 in Domain II demonstrated that the aromatic residues play an important role GPI-anchored receptor Tyr-1 binding [23]. Similarly, aromatic residues (Tyr42, Tyr43, Tyr49, Tyr209, and Phe212) of epsilon have been explicated as putative binding determinants with its receptors [24]. In Cry51Aa1, Domain I has a large cluster of solvent-accessible aromatic residues in the region spanning Tyr200 to Phe235: Tyr200, Tyr203, Tyr216, Trp228, and Phe235 are located in loops regions while Tyr19, Trp20, Trp208, Trp223, and Tyr224 are located in a-helices or $\beta$-strands. As aromatic rings often stack against the planar faces of sugar pyranose rings, tryptophan and tyrosine rings are considered to provide hydrophobic driving forces towards carbohydrate moieties of the receptors [25]. These aromatic clusters may be potential sources of carbohydrate-protein interactions such as ligand binding or receptor recognition. Additionally, aromatic residues may contribute to the stability of Domain I by forming intramolecular hydrogen bonds to the side chains of neighboring residues (Fig. S2).

\subsection{Abundant serine and threonine residues}


Almost of a quarter (23.3\%) of the Cry51Aa1 amino acid composition is either serine or threonine. Interestingly, these residues are dominant in three regions of the structure. (1) A Ser/Thr patch located in the middle of amphipathic $\beta$-hairpin, close to the Domain II-III interface; (2) Ser/Thr clusters that flanked the $\beta 6-\beta 7$ hairpin located in strand $\beta 5$ and $\beta 5$, and also in the loops connecting to the $\beta 6-\beta 7$ hairpin; (3) A Ser/Thr cluster associated with strands $\beta 2, \beta 9$ and $\beta 12$ at the Domain I-II interface. The hydroxyl groups of Ser/Thr residues are capable of forming intramolecular hydrogen bonds (in addition to the inter-strand main chain hydrogen bonding) to the adjacent strands or helices, and provide specific but strong forces to build up a hydrogen bond network as to further stabilize the molecule (Fig. 4).

With respect to other function of Ser/Thr clusters, three possible roles have been suggested, (1) allowing the molecule to move freely and closely to the membrane when $\mathrm{N}$-terminal domain binding to the receptor [6]; (2) guiding the amphipathic loop towards the membrane [26]; and (3) promoting oligomerization [24]. As lacking experimental evidence of Cry51Aa1 interacting with receptors on the membrane, it is difficult to ascertain which kind of interaction is involved between the Ser/Thr track and membrane during the transmembrane procedure. However, based on the expectation of abundant Ser/Thr residues that could be exposed to the solvent after the amphipathic $\beta 6-\beta 7$ hairpin stretching out, it seems more possible for these Ser/Thr residues to drive the molecule towards neighboring monomer by forming intermolecular hydrogen bonds between the $\beta$-strands that flanked amphipathic $\beta$-hairpins. So that the Ser/Thr related interactions could promote oligomerization and also help to stabilize the complex.

\subsection{Glycine-rich loop on the amphipathic $\beta$-hairpin}


The amphipathic $\beta$-hairpin is one the most remarkable features in the aerolysintype $\beta$-PFTs. Although this amphipathic region shows varied architecture within the family, it would finally undergo a conformational change into a transmembrane $\beta$ hairpin when penetrating the membrane. In Cry51Aa1, an amphipathic $\beta$-hairpin formed by strands $\beta 6$ and $\beta 7$ is also observed. The loop connecting the two strands is glycine rich ${ }^{127}$ GEGGG $^{131}$ and shows little sequence conservation to other aerolysin-type $\beta$-PFTs. The glycine-rich loop is anchored to helix a5 and the Cterminal tail via hydrogen bonding between Glu128 N and Val297 O and ASN133 ND2 and ASN296 O (Fig 3E). Based on the current thinking that the $\beta$-hairpin is involved in membrane insertion, it is envisioned that upon proteinase activation, the C-terminal tail would be removed and the strong interactions it provided in stabilizing the dimer would be lost, which would disrupt the dimer and untether the glycine-rich loop. Once the glycine-rich loop acquires adequate flexibility, this frontend is most likely to lead the amphipathic $\beta$-hairpin to stretch out. As the smallest amino acid, glycine has greater conformational freedom than other amino acids due to its unique dihedral angles. So that, in the membrane binding or transmembrane process, it can provide flexible conformational change on the backbone and favor hydrophobic or hydrophilic environment. Particularly, during oligomerization, the aggregated glycine-rich loops from each monomers may gain enhanced conformational flexibility than other amino acids, which will subsequently contribute to the overall hydrophobic features of transmembrane $\beta$-barrel and guides the amphipathic $\beta$-hairpins penetrating through the lipid bilayer.

Interestingly, a glycine-rich loop located at the amphipathic $\beta$-hairpin was found in Staphylococcus aureus $\beta$-PFTs family. As described in a-haemolysin (PDB ID 
7AHL), a glycine-rich loop ( $\mathrm{Gly}^{119}-\mathrm{Gly}^{143}$ ) was considered to be exposed to the solvent in a monomer and pre-pore oligomer state, but project into lumen during transmembrane insertion of the heptamer [27]. While the pore-forming mechanisms of a-haemolysin and aerolysin-type $\beta$-PFTs are dissimilar, evidence of the glycine-rich loop from a-haemolysin oligomers presents similar characteristics with Cry51Aa1 $\beta 6-\beta 7$ loop, and provides a good comparative reference for future studies on the transmembrane process.

In addition, next to the glycine-rich loop, there is an aromatic residue Phe141 and a hydrophobic region $\left({ }^{123} \mathrm{IPFI}^{126}\right.$ ) at strand $\beta 7$ and $\beta 6$, respectively. Aromatic residues are considered to anchor the transmembrane $\beta$-barrel to the lipid bilayer, such as the aromatic belt of the swirling membrane-insertion model [26]. Moreover, in the rivet-like model of aerolysin, the hydrophobic tip was demonstrated with the capability of directing membrane insertion [28]. It could be speculated that the hydrophobic region of Cry51Aa1 could effectively enhance the leading activity of the glycine-rich loop when penetrating the lipid bilayer, while the aromatic ring at the $\beta$-barrel plays a role in oligomer stabilization.

In summary, we present a $1.65 \AA$ resolution crystal structure of Cry51Aa1 toxin. With the significant structural similarity, Cry51Aa1, or more broadly the Cry51A toxins, would be a new insecticidal toxins group belonging to the aerolysin-type $\beta$ PFTs. As to its mode of toxic action, Cry51Aa1 shares several important features with other aerolysin-type $\beta$-PFTs, a three domain architecture, a long amphipathic $\beta$-hairpin with a glycine-rich loop, which suggest a possible mode of membrane intercalation that warrants further study. 


\section{Acknowledgement}

This project was supported by the State Key Program of National Natural Science

of China (Grant No. 30930004) and from the Georgia Research Alliance. Use of the Advanced Photon Source was supported by the U. S. Department of Energy, Office of Science, Office of Basic Energy Sciences, under Contract No. W-31-109-Eng-38."

Data were collected at Southeast Regional Collaborative Access Team (SER-CAT) 22-BM beamline at the Advanced Photon Source, Argonne National Laboratory.

Supporting institutions may be found at www.ser-cat.org/members.html. 


\section{References}

[1] A.I. Aronson, W. Beckman, P. Dunn, Bacillus thuringiensis and related insect pathogens, Microbiol Rev. 50 (1986) 1-24.

[2] E. Schnepf, N. Crickmore, J. Van Rie, D. Lereclus, B. Baum, J. Feitelson, D.R. Zeigler, D.H. Dean, Bacillus thuringiensis and its pesticidal crystal proteins, Microbiol. Mol. Biol. Rev. 62 (1998) 775806.

[3] M.J. Adang, N. Crickmore, J.L. Jurat-Fuentes, Diversity of Bacillus thuringiensis crystal toxins and mechanism of action, in: S.D. Tarlochan, S.G. Sarjeet (Eds.), Adv. Insect. Physiol., Academic Press, Oxford, 2014, pp. 39-87.

[4] C. Xu, B.C. Wang, Z. Yu, M. Sun, Structural insights into Bacillus thuringiensis Cry, Cyt and parasporin toxins, Toxins. 6 (2014) 2732-2770.

[5] E. Mizuki, Y.S. Park, H. Saitoh, S. Yamashita, T. Akao, K. Higuchi, M. Ohba, Parasporin, a human leukemic cell-recognizing parasporal protein of Bacillus thuringiensis, Clin. Diagn. Lab. Immunol. 7 (2000) 625-634.

[6] T. Akiba, Y. Abe, S. Kitada, Y. Kusaka, A. Ito, T. Ichimatsu, H. Katayama, T. Akao, K. Higuchi, E. Mizuki, M. Ohba, R. Kanai, K. Harata, Crystal structure of the parasporin-2 Bacillus thuringiensis toxin that recognizes cancer cells, J. Mol. Biol. 386 (2009) 121-133.

[7] T. Akiba, K. Higuchi, E. Mizuki, K. Ekino, T. Shin, M. Ohba, R. Kanai, K. Harata, Nontoxic crystal protein from Bacillus thuringiensis demonstrates a remarkable structural similarity to pore-forming toxins, Proteins: Struct., Funct., Bioinf. 63 (2006) 243-248.

[8] M.S. Kelker, C. Berry, S.L. Evans, R. Pai, D.G. McCaskill, N.X. Wang, J.C. Russell, M.D. Baker, C. Yang, J.W. Pflugrath, M. Wade, T.J. Wess, K.E. Narva, Structural and biophysical characterization of Bacillus thuringiensis insecticidal proteins Cry34Ab1 and Cry35Ab1, PLoS One. 9 (2014) e112555.

[9] D.F. Huang, J. Zhang, F.P. Song, Z.H. Lang, Microbial control and biotechnology research on Bacillus thuringiensis in China, J. Invertebr. Pathol. 95 (2007) 175-180.

[10] D. Peng, Y. Luo, S. Guo, H. Zeng, S. Ju, Z. Yu, M. Sun, Elaboration of an electroporation protocol for large plasmids and wild-type strains of Bacillus thuringiensis, J. Appl. Microbiol. 106 (2009) 18491858.

[11] Z. Otwinowski, W. Minor, Processing of X-ray diffraction data collected in oscillation mode, Methods Enzymol. 276 (1997) 307-326.

[12] P.D. Adams, P.V. Afonine, G. Bunkoczi, V.B. Chen, I.W. Davis, N. Echols, J.J. Headd, L.W. Hung, G.J. Kapral, R.W. Grosse-Kunstleve, A.J. McCoy, N.W. Moriarty, R. Oeffner, R.J. Read, D.C. Richardson, J.S. Richardson, T.C. Terwilliger, P.H. Zwart, PHENIX: a comprehensive Python-based system for macromolecular structure solution, Acta. Crystallogr. D. Biol. Crystallogr. 66 (2010) 213-221.

[13] S. Panjikar, V. Parthasarathy, V.S. Lamzin, M.S. Weiss, P.A. Tucker, Auto-Rickshaw: an automated crystal structure determination platform as an efficient tool for the validation of an X-ray diffraction experiment, Acta. Crystallogr. D. Biol. Crystallogr. 61 (2005) 449-457.

[14] P. Emsley, B. Lohkamp, W.G. Scott, K. Cowtan, Features and development of Coot, Acta. Crystallogr. D. Biol. Crystallogr. 66 (2010) 486-501.

[15] B.W. Matthews, Solvent content of protein crystals, J. Mol. Biol. 33 (1968) 491-497.

[16] Y. Park, G. Hua, M.A.F. Abdullah, K. Rahman, M.J. Adang, Cadherin fragments from Anopheles gambiae synergize Bacillus thuringiensis Cry4Ba's toxicity against Aedes aegypti Larvae, Appl. Environ. Microbiol. 75 (2009) 7280-7282.

[17] I. Corp., IBM SPSS statistics for Windows, Version 22.0., IBM Corp., Amonk, NY, 2013.

[18] E. Krissinel, K. Henrick, Inference of macromolecular assemblies from crystalline state, J. Mol. Biol 372 (2007) 774-797.

[19] L. Holm, R. P, Dali server: Conservation mapping in 3D, Nucl. Acids Res. 38 (2010) W545-549. 
[20] J.A. Baum, U.R. Sukuru, S.R. Penn, S.E. Meyer, S. Subbarao, X. Shi, S. Flasinski, G.R. Heck, R.S. Brown, T.L. Clark, Cotton plants expressing a hemipteran-active Bacillus thuringiensis crystal protein impact the development and survival of Lygus hesperus (Hemiptera: Miridae) nymphs, J. Econ. Entomol. 105 (2012) 616-624.

[21] J. Baum, S.R. Penn, S. Flasinski, X. Shi, G.R. Heck, S.U. Rao, Hemipteran- and coleopteran- active toxin proteins from Bacillus thuringiensis U.S. Patent 8513 493, issued date Aug 20, 2013.

[22] L. Abrami, M.C. Velluz, Y. Hong, K. Ohishi, A. Mehlert, M. Ferguson, T. Kinoshita, F.G. van der Goot, The glycan core of GPI-anchored proteins modulates aerolysin binding but is not sufficient: the polypeptide moiety is required for the toxin-receptor interaction, FEBS Lett. 512 (2002) 249-254.

[23] C.R. Mackenzie, T. Hirama, J.T. Buckley, Analysis of receptor binding by the channel-forming toxin aerolysin using surface plasmon resonance, J. Biol. Chem. 274 (1999) 22604-22609.

[24] A.R. Cole, M. Gibert, M. Popoff, D.S. Moss, R.W. Titball, A.K. Basak, Clostridium perfringens epsilontoxin shows structural similarity to the pore-forming toxin aerolysin, Nat. Struct. Mol. Biol. 11 (2004) 797-798.

[25] J.L. Asensio, A. Ardá, F.J. Cañada, J. Jiménez-Barbero, Carbohydrate-aromatic interactions, Acc. Chem. Res. 46 (2013) 946-954.

[26] M.T. Degiacomi, I. Lacovache, L. Pernot, M. Chami, M. Kudryashev, H. Stahlberg, F.G. van der Goot, M. Dal Peraro, Molecular assembly of the aerolysin pore reveals a swirling membrane-insertion mechanism, Nat. Chem. Biol. 9 (2013) 623-629.

[27] B. Walker, O. Braha, S. Cheley, H. Bayley, An intermediate in the assembly of a pore-forming protein trapped with a genetically-engineered switch, Chem. Biol. 2 (1995) 99-105.

[28] I. lacovache, P. Paumard, H. Scheib, C. Lesieur, N. Sakai, S. Matile, M.W. Parker, F.G. van der Goot, A rivet model for channel formation by aerolysin-like pore-forming toxins, EMBO J. 25 (2006) 457466. 
Fig.1. Ribbon representation of Cry51Aa1 monomer. Secondary structure of ahelices and $\beta$-strands are presented in yellow and cyan, respectively. The amphipathic $\beta$-hairpin is shown in magenta.

Fig.2. Structure comparison of Cry51Aa1 and its structural homologs of aerolysintype proteins with the amphipathic $\beta$-hairpin. (A) Structural conservation of aerolysin-type proteins, from left to right: Cry51Aa1, 26NPT (PDB ID 2D42), epsilon (PDB ID 1UYJ), and parasporin-2 (PDB ID 2ZTB). Unconserved a-helix and $\beta$-strand are colored in grey. (B) Primary sequence alignment of Cry51Aa1, Cry51Aa2 (GeneBank: ADK94873.1), 26NPT, epsilon (ETX) and parasporin-2 (PS2) by Promals3D. Cylinders and arrows above the sequence indicate helical and $\beta$-strand conformations of Cry51Aa1. Aromatic residues and Ser/Thr residues are colored in red and blue, respectively, while conserved regions are presented in grey background, with the strictly conserved residues marked with black triangle at the bottom.

Fig.3. Intramolecular and intermolecular interactions of Cry51Aa1 molecule. (A) Surface representation of a Cry51Aa1 dimer viewed along a crystallographic 2-fold axis. (B) Side view of the dimer. (C) Calculated B-factors of Cry51Aa1 monomer. Lowest B-factor areas located at domain boundaries are presented in purple blue. (D) Monomer-monomer interactions at dimer interface. (E) Intramolecular interactions around glycine-rich loop at Domain II and Domain III boundary. Hydrogen bonds at both dimer and inter-domain interfaces are indicated in red dashed lines. 
Fig.4. Ser/Thr residues involved stabilizing interactions. Hydrogen bonds generated by Ser/Thr residues adjacent to the amphipathic $\beta$-hairpin $(A)$ and at the boundary of Domain I and Domain II (B) are presented in brown dashed lines. Ser/Thr residues are highlighted in green. 


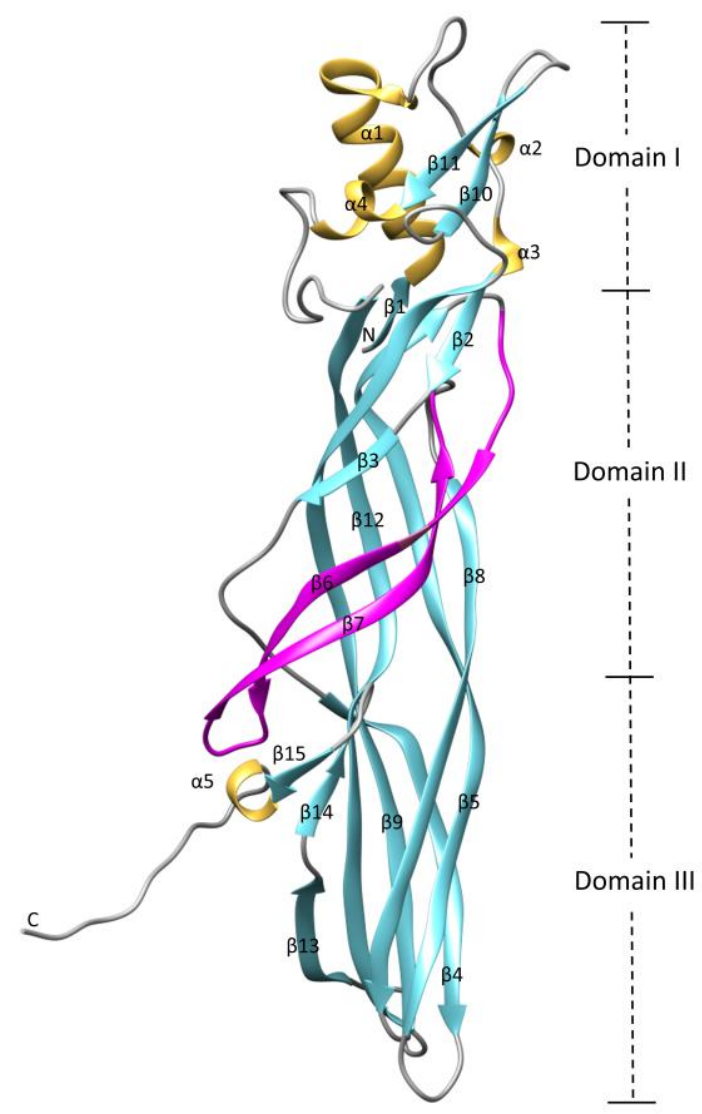

Figure 1 
A
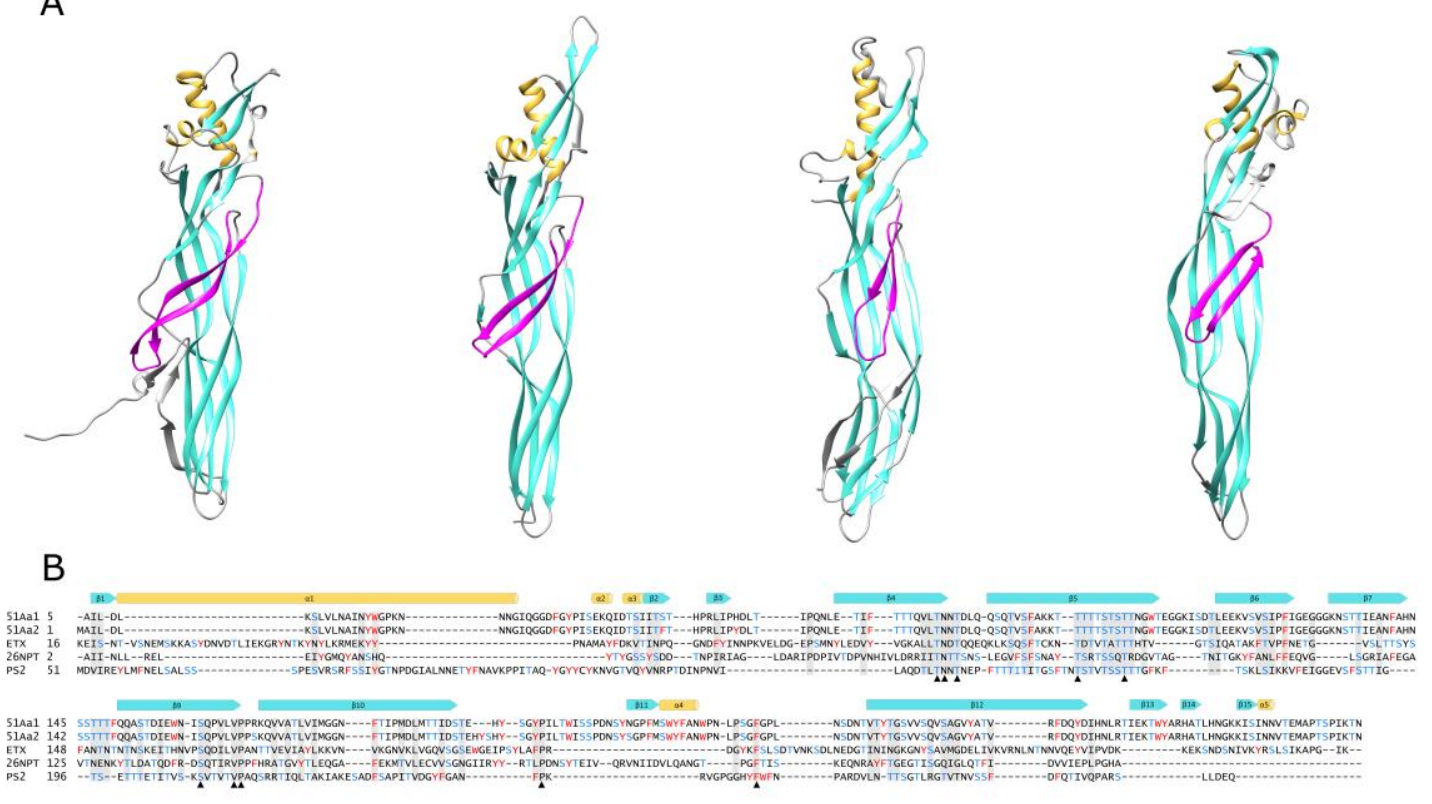

Figure 2 


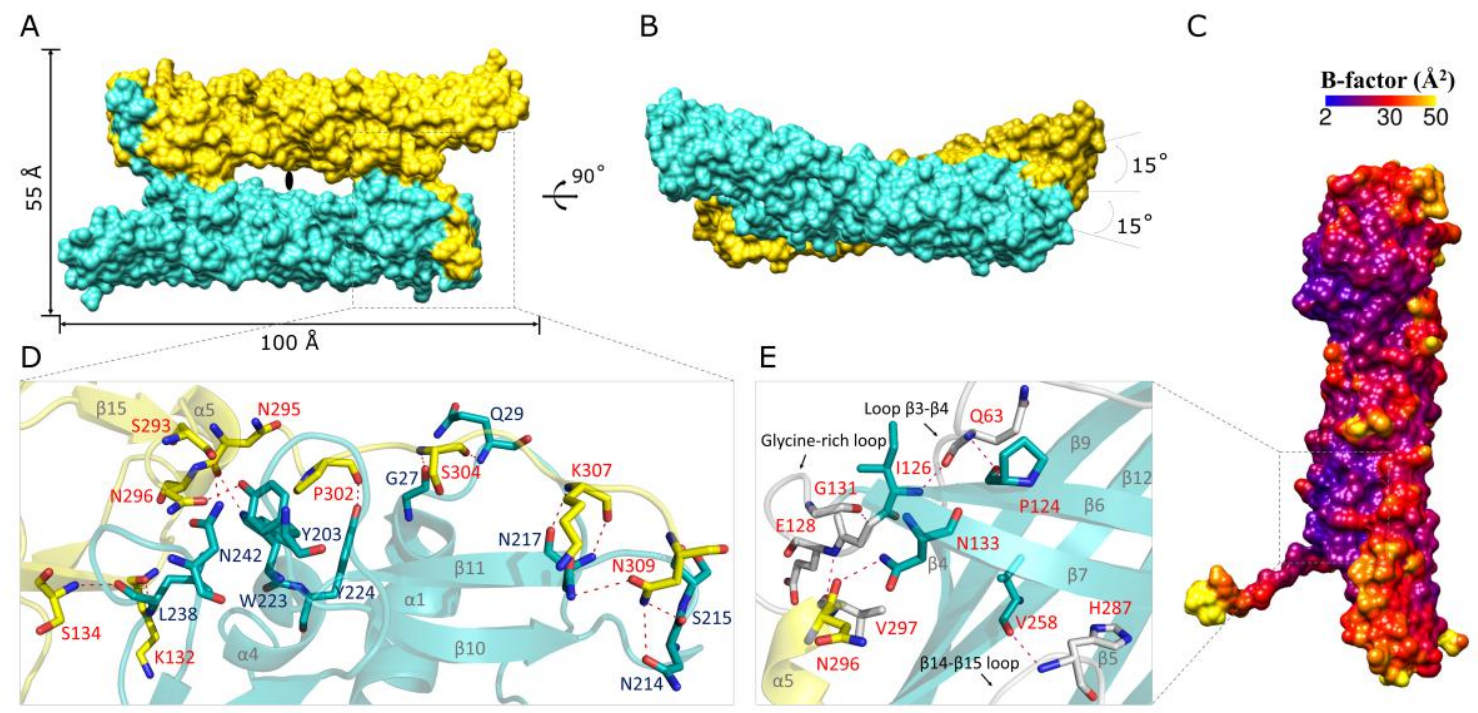

Figure 3 

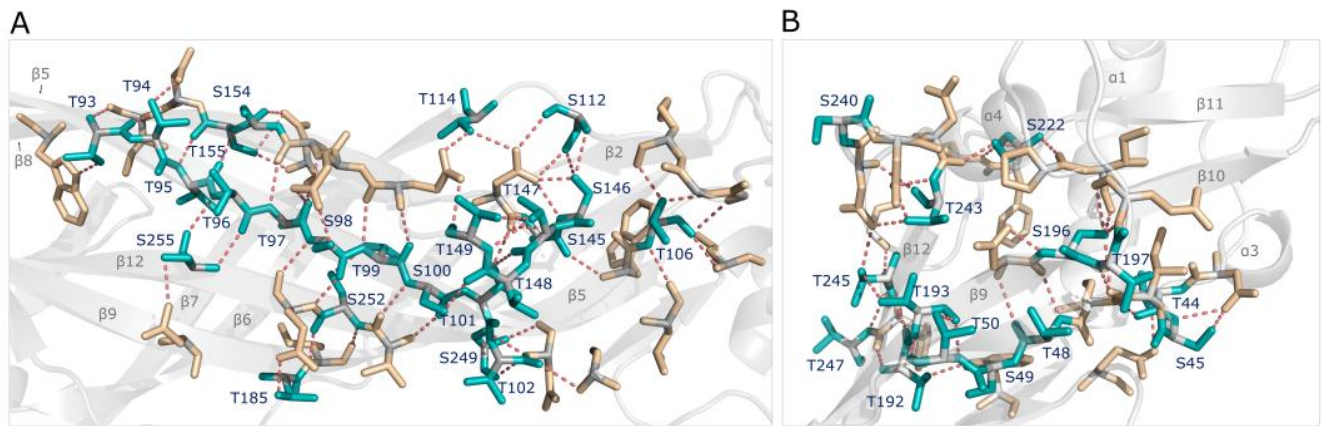

Figure 4 